\title{
THE $p$-SYSTEM II: THE VACUUM
}

\author{
ROBIN YOUNG \\ Department of Mathematics \& Statistics \\ University of Massachusetts \\ E-mail: young@math.umass.edu
}

\begin{abstract}
We consider the equations of isentropic gas dynamics in Lagrangian coordinates. We are interested in global interactions of large waves, and their relation to global solvability and well-posedness for large data. One of the main difficulties in this program is the possible occurrence of a vacuum, in which the specific volume is infinite. In this paper we show that the vacuum cannot be generated in finite time. More precisely, if the vacuum is present for some positive time, then it must be present in the initial data, in a precise sense which is given. We also discuss the annihilation of vacuums that are present in the initial data.
\end{abstract}

1. Introduction. We consider the equations of isentropic gas dynamics in Lagrangian coordinates,

$$
\left(\begin{array}{l}
v \\
u
\end{array}\right)_{t}+\left(\begin{array}{c}
-u \\
p(v)
\end{array}\right)_{x}=0,
$$

where $u$ is the fluid velocity, $v=1 / \rho$ is specific volume, $p$ is the pressure. Our ultimate goal is the extension of the Glimm-Lax theory to include waves of arbitrary strength [5]. We develop techniques that can be extended to many larger systems, of which the $p$-system is a subsystem [16]. The main new feature of our analysis is the exact treatment of waves and their interactions, in place of asymptotic estimates for interactions which are generally valid only for weak waves $[4,13]$.

One of the main difficulties in dealing with large data is the possible occurrence of the vacuum. At the vacuum, the specific volume $v$ becomes infinite and must be taken to be a measure. Thus the solution is not stable in $B V_{\text {loc }}$, and indeed Glimm's asymptotic interaction estimates fail near the vacuum [7]. Our purpose in this paper is to fully understand the circumstances of vacuum formation, and to study the effects of wave interactions on the vacuum.

This work was partly supported by the USA's NSF under grant number DMS-0104485. 2000 Mathematics Subject Classification: 35L65, 76N15.

The paper is in final form and no version of it will be published elsewhere. 
A necessary condition for the system to admit the vacuum state is that the integral of the sound speed converges as the specific volume goes to infinity,

$$
\int_{1}^{\infty} c(\nu) d \nu<\infty
$$

where the sound speed is defined by

$$
c^{2}(v)=-p^{\prime}(v) .
$$

This is true for the most interesting physical systems, and we assume it throughout this paper. We also assume for convenience that the pressure is convex, $p^{\prime \prime}(v)>0$. However, this assumption is not essential, and our methods extend directly to nonconvex pressures, see $[15]$ and also $[2,14]$.

It is well known that a vacuum can form in the solution to the Riemann problem [8]. Physically, the difference between the left and right velocities is so great that the gas cannot expand sufficiently, and a vacuum is formed. By analogy, a vacuum will form in a general solution if the data has a jump similar to that in which the Riemann solution contains a vacuum. Our goal here is to show that this is the only way for a vacuum to form: in other words, if a vacuum is present at some finite time $t>0$, then it must have come from a large jump in the initial data, and been formed at time $t=0+$.

Our strategy is elementary: we first identify which wave interactions can give rise to the vacuum state. We show that any interaction of a shock and another wave moves us further from the vacuum: indeed, the sound speed is greater, and thus the specific volume is smaller, behind a shock (i.e. for later times). Therefore the only interaction that can generate a vacuum is that of a forward and backward rarefaction. We then analyze the region in spacetime in which two rarefactions interact, and calculate how long it takes them to pass through each other. We find that the time it takes to generate the vacuum is infinite. Indeed, the vacuum can be characterized by vanishing sound speed, so as the sound speed approaches zero, the time of interaction grows without bound, and the vacuum is never reached in finite time.

Our construction is based on the global solution to the Riemann problem developed in [15]. We develop a concise description of the nonlinear waves in terms of a single function of two variables. Thus we have

$$
u_{r}-u_{l}=g\left(v_{a}, v_{b}\right),
$$

where the subscripts refer to left, right, ahead and behind, respectively, and $g$ is a given function. We combine forward and backward waves to solve the Riemann problem, in which the waves can have arbitrary strength. The following was proved in [15].

THEOREM 1. Given constant left and right states $\left(\begin{array}{lll}v_{l} & u_{l}\end{array}\right)^{t}$ and $\left(\begin{array}{ll}v_{r} & u_{r}\end{array}\right)^{t}$, respectively, there is a unique solution to the corresponding Riemann problem, provided that the condition

$$
u_{r}-u_{l}<g\left(v_{l}, \infty\right)+g\left(v_{r}, \infty\right)
$$

holds. Moreover, the intermediate state $\left(v_{*} u_{*}\right)^{t}$ (and hence wave strength) is a $C^{2}$ function of the data. 
The failure of this one-sided inequality implies the appearance of a vacuum. Here $v$ becomes a Radon measure, the singular part of which is supported on the vacuum region, which for the Riemann problem is the positive $t$-axis. Since the gas rarefies on either side of the vacuum, the velocity $u$ increases from left to right, with a positive jump across the $t$-axis. The following is also proved in [15].

THEOREM 2. Given arbitrary states $\left(v_{l} u_{l}\right)^{t}$ and $\left(v_{r} u_{r}\right)^{t}$ for which (1.5) fails, there is a unique solution $(v(x, t), u(x, t))^{t}$ to the Riemann problem, where the velocity

$$
u(x, t) \in L^{\infty} \cap B V \cap L_{\mathrm{loc}}^{1}
$$

is a bounded increasing function, while $v(x, t)$ is a Radon measure. Moreover this solution is Lipschitz continuous in time as a distribution in $L_{\text {loc }}^{1}$.

By a simple stability argument, we see that a vacuum will develop in a general solution if the Cauchy data has a jump and whose left and right limits violate (1.5). The time of existence of this vacuum depends on the number and size of shocks approaching the vacuum [7]. We reprove in this context the fact that a shock will meet the vacuum in finite time, at which time the shock disappears and the strength of the vacuum is diminished.

We treat all interactions exactly with the use of (1.4). Thus resolving the incoming and outgoing states, the general forward-backward interaction is described by

$$
g\left(v_{l}, v_{*}\right)+g\left(v_{r}, v_{*}\right)=g\left(v_{m}, v_{l}\right)+g\left(v_{m}, v_{r}\right),
$$

where again there is no restriction on the size of the waves. This may not always have a solution, meaning that the result of the interaction will contain a vacuum. Restricting ourselves to this case, we consider the interaction of two rarefactions. Since the boundary of the interaction region is characteristic [3], we calculate the path of an opposite characteristic across a rarefaction wave. Eliminating $x$, we obtain a differential equation for $t$, which we solve to get in the simplest case

$$
\sqrt{c} t=\sqrt{c_{0}} t_{0}
$$

so that as the sound speed $c \rightarrow 0, t \rightarrow \infty$ and the time of passage through the interaction is unbounded. Thus we have the following theorem.

THEOREM 3. A vacuum cannot form from wave interactions in finite time. Thus any vacuum that appears in the solution must be present in the initial data. In particular, if the initial velocity is continuous, there is no vacuum in the solution.

Liu and Smoller identify two different types of vacuum, namely compression and rarefaction vacuums [7]. The vacuum which develops at $t=0+$ is always a rarefaction vacuum. Left alone, rarefaction vacuums will persist for all times, expanding at a constant rate in physical space. However, a shock which approaches the vacuum (i.e. enters the adjacent rarefaction) will meet the vacuum in finite time. The shock disappears when it meets the vacuum, while the vacuum is weakened by the shock. By this we mean that the weight on the delta-function identifying the vacuum decreases, and may become negative. A negative weight implies that the vacuum is now a compression vacuum, so its width decreases in physical space, and it will be extinguished in finite time. Our results are consistent with [7], as is expected [10]. 
In the following sections, we recall the solution of the Riemann problem, and describe the condition for the vacuum to be embedded in the initial data. We next consider wave interactions, and show that the vacuum cannot form from wave interactions in finite time. Finally we discuss the effect of shocks meeting the vacuum, and describe the possible annihilation of the vacuum. In concluding, we describe our approach to extending the existence theory and map out the next steps in our program.

2. The Riemann problem. We briefly recall the construction of the solution to the Riemann problem which was constructed in [15]. For simplicity we assume the pressure is convex, although our methods generalize directly to the nonconvex case.

The quasilinear form of our system is

$$
\left(\begin{array}{l}
v \\
u
\end{array}\right)_{t}+A(v)\left(\begin{array}{l}
v \\
u
\end{array}\right)_{x}=0
$$

where $A(v)$ is the flux matrix

$$
A(v)=\left(\begin{array}{cc}
0 & -1 \\
p^{\prime}(v) & 0
\end{array}\right)=\left(\begin{array}{cc}
0 & -1 \\
-c^{2}(v) & 0
\end{array}\right) .
$$

The characteristic wavespeeds are the eigenvalues $\pm c(v)$, and the rarefaction curves are the integrals of the eigenvectors. In a rarefaction, we have

$$
\frac{d x}{d t}= \pm c(v(x, t))
$$

while across the wave,

$$
\frac{d}{d \epsilon}\left(\begin{array}{l}
v \\
u
\end{array}\right)=\left(\begin{array}{c}
1 \\
\mp c(v)
\end{array}\right) .
$$

Integrating, we get

$$
u-u_{0}=\mp \int_{v_{0}}^{v} c(\nu) d \nu
$$

where $v$ parameterizes the curve. The correct branch of this curve is that for which the sound speed is greater ahead of the wave.

The shocks are given by the Rankine-Hugoniot conditions [6, 9],

$$
\sigma[v]=-[u] \quad \text { and } \quad \sigma[u]=[p(v)]
$$

subject to the entropy inequality, which states that the sound speed is greater behind a shock. The solution to $(2.5)$ is easily seen to be

$$
u-u_{0}=-\sigma\left(v_{0}, v\right)\left(v-v_{0}\right),
$$

where

$$
\sigma\left(v_{0}, v\right)= \pm \sqrt{\frac{p\left(v_{0}\right)-p(v)}{v-v_{0}}},
$$

and $v$ again parameterizes the curve. 
We now combine these waves and solve the Riemann problem. First, define the function $g: \mathbf{R}^{2} \rightarrow \mathbf{R}$ by

$$
g\left(v_{1}, v_{2}\right)= \begin{cases}\int_{v_{1}}^{v_{2}} c(\nu) d \nu, & \text { for } \quad v_{1} \leq v_{2}, \\ -\sqrt{\left(p\left(v_{2}\right)-p\left(v_{1}\right)\right)\left(v_{1}-v_{2}\right)}, & \text { for } \quad v_{1} \geq v_{2} .\end{cases}
$$

The forward and backward wave curves can now be described by

$$
u_{r}-u_{l}=g\left(v_{a}, v_{b}\right),
$$

where the subscripts $l$ and $r$ refer to the left and right states, while $a$ and $b$ refer to the states ahead of and behind the wave, respectively (so $a=l$ for a backward wave). As expected, the wave is a shock if the sound speed behind the wave is faster,

$$
c\left(v_{a}\right)<c\left(v_{b}\right), \text { that is } v_{a} \geq v_{b},
$$

while the opposite inequalities hold for rarefactions. Note that this description holds exactly for all elementary waves of arbitrary strength. Equation (2.8) also holds for composite waves when the pressure in nonconvex provided $g$ is appropriately defined, see $[15,2]$.

We combine these descriptions of forward and backward waves to solve the Riemann problem with arbitrary left and right states. Given two states $\left(v_{l} u_{l}\right)^{t}$ and $\left(v_{r} u_{r}\right)^{t}$, we look for a middle state $\left(v_{*} u_{*}\right)^{t}$, so that a backward wave joins $\left(v_{l} u_{l}\right)^{t}$ to $\left(v_{*} u_{*}\right)^{t}$, and a forward wave joins $\left(v_{*} u_{*}\right)^{t}$ to $\left(v_{r} u_{r}\right)^{t}$.

According to $(2.8)$, if $\left(v_{l} u_{l}\right)^{t}$ is joined to $\left(v_{*} u_{*}\right)^{t}$ by a backward wave, then we have

$$
u_{*}-u_{l}=g\left(v_{l}, v_{*}\right),
$$

while if $\left(v_{*} u_{*}\right)^{t}$ is joined to $\left(v_{r} u_{r}\right)^{t}$ by a forward wave, then

$$
u_{r}-u_{*}=g\left(v_{r}, v_{*}\right) \text {. }
$$

Eliminating $u_{*}$, we get the equation

$$
u_{r}-u_{l}=g\left(v_{l}, v_{*}\right)+g\left(v_{r}, v_{*}\right) \equiv \phi\left(v_{*}\right),
$$

and we wish to solve for $v_{*}$. In [15] it is shown that $\phi$ is an increasing function whose range is unbounded below, so that (2.9) is uniquely solvable provided $u_{r}-u_{l}$ is not too large.

THEOREM 1. Given constant left and right states $\left(\begin{array}{lll}v_{l} & u_{l}\end{array}\right)^{t}$ and $\left(\begin{array}{ll}v_{r} & u_{r}\end{array}\right)^{t}$, respectively, there is a unique solution to the corresponding Riemann problem, provided that the condition

$$
u_{r}-u_{l}<g\left(v_{l}, \infty\right)+g\left(v_{r}, \infty\right)
$$

holds. Moreover, the intermediate state $\left(v_{*} u_{*}\right)^{t}$ (and hence wave strength) is a $C^{2}$ function of the data.

2.1. Properties of $g$. We now enumerate some properties of the function $g$ which will be useful in the sequel. Across a rarefaction, $g$ is the integrated wavespeed,

$$
g\left(v_{1}, v_{2}\right)=\int_{v_{1}}^{v_{2}} c(\nu) d \nu=h\left(v_{2}\right)-h\left(v_{1}\right)
$$


where

$$
h(v)=\int_{1}^{v} c(\nu) d \nu
$$

provided $v_{1} \leq v_{2}$. We can interpret $g$ in the same way across a shock, if we write for $v_{1} \leq v_{2}$,

$$
g\left(v_{1}, v_{2}\right)=\sqrt{\frac{p\left(v_{2}\right)-p\left(v_{1}\right)}{v_{1}-v_{2}}}\left(v_{2}-v_{1}\right)=\int_{v_{1}}^{v_{2}} \sigma\left(v_{1}, v_{2}\right) d \nu
$$

where $\sigma$ is the (absolute) shock speed. Here $-\sigma^{2}$ is the slope of the secant on the graph of $p(v)$, which is also the tangent of the upper convex envelope of $p$ between $v_{1}$ and $v_{2}$, which we denote by $p^{\cap}$. It is convenient to write

$$
g\left(v_{1}, v_{2}\right)=h\left(v_{2}\right)-h\left(v_{1}\right)+\theta\left(v_{1}, v_{2}\right)
$$

where $\theta\left(v_{1}, v_{2}\right)$ is a measure of the difference between shock and rarefaction curves. Indeed, we have

$$
\theta\left(v_{1}, v_{2}\right)=0 \quad \text { if } \quad v_{1} \leq v_{2}
$$

and

$$
\theta\left(v_{1}, v_{2}\right)=\int_{v_{1}}^{v_{2}} \sqrt{-p^{\cap^{\prime}}}-\sqrt{-p^{\prime}} d \nu<0, \quad \text { for } \quad v_{1}>v_{2} .
$$

It is shown in [11] that the function $\theta$ is decreasing in the first variable and increasing in the second,

$$
\theta_{; 1}\left(v_{1}, v_{2}\right)<0 \text { and } \theta_{; 2}\left(v_{1}, v_{2}\right)>0
$$

It is now clear that $g$ is a $C^{2}$ function, also monotone decreasing in the first variable and increasing in the second, and that the relations

$$
\begin{aligned}
& v_{1}>v_{2}, \quad h\left(v_{1}\right)>h\left(v_{2}\right), \\
& g\left(v_{1}, v_{2}\right)<0, \quad \text { and } \theta\left(v_{1}, v_{2}\right)<0
\end{aligned}
$$

are equivalent, and identify the presence of a shock.

3. The vacuum. If the velocity difference $u_{r}-u_{l}$ of the Riemann data is too large, no finite intermediate state $v_{*}$ exists, and a vacuum develops. Physically, the gas rarefies as much as it can to the right of the left state $\left(v_{l} u_{l}\right)^{t}$, filling the wedge

$$
-c\left(v_{l}\right) t \leq x<0,
$$

inside which we have

$$
\begin{gathered}
c(v(x, t))=-x / t \\
u(x, t)-u_{l}=g\left(v_{l}, v(x, t)\right)=\int_{v_{l}}^{v(x, t)} c(\nu) d \nu,
\end{gathered}
$$

so that the extreme state on the inside edge of this wedge is

$$
v(0-, t)=\infty \quad \text { and } \quad u(0-, t)=u_{-} \equiv u_{l}+g\left(v_{l}, \infty\right) .
$$


Similarly, the gas rarefies to the left of $\left(v_{r} u_{r}\right)^{t}$, giving the extreme state

$$
v(0+, t)=\infty \quad \text { and } \quad u(0+, t)=u_{+} \equiv u_{r}-g\left(v_{r}, \infty\right),
$$

where we have $u_{-} \leq u_{+}$by the failure of (2.10).

In Lagrangian coordinates, the vacuum has no width, and the coordinate transformation from Eulerian coordinates degenerates. The resolution of this difficulty consists in allowing the specific volume $v$ to be a Radon measure, while the other variables remain bounded (and indeed density, pressure and sound speed vanish). The singular part of this Radon measure is supported on the $t$-axis, and thus has the form

$$
\nu_{S}=w(t) \delta(x),
$$

where $w(t)$ is a bounded function. Indeed, taking $w(0)=0$, and solving the equation

$$
v_{t}-u_{x}=0
$$

in the distributional sense yields

$$
\nu_{S}=\left(u_{+}-u_{-}\right) t \delta(x) .
$$

We thus have the following theorem, also proved in [15].

THEOREM 2. Given arbitrary states $\left(v_{l} u_{l}\right)^{t}$ and $\left(v_{r} u_{r}\right)^{t}$ for which (2.10) fails, there is a unique solution $(v(x, t), u(x, t))^{t}$ to the Riemann problem, where the velocity

$$
u(x, t) \in L^{\infty} \cap B V \cap L_{\text {loc }}^{1}
$$

is a bounded increasing function, while $v(x, t)$ is a Radon measure whose singular part is the Dirac measure (3.3) and whose absolutely continuous part is locally integrable. Moreover this solution is Lipschitz continuous in time as a distribution in $L_{\text {loc }}^{1}$.

3.1. Vacuum in the data. We now consider the possible formation of the vacuum for general data. We assume here that the initial specific volume is finite,

$$
v(x, 0)=v_{0}(x)<\infty \quad \text { so }\left(v_{0} u_{0}\right)^{t} \in L^{\infty},
$$

where we have redefined $v_{0}$ on a set of measure zero as necessary. It is convenient to assume in fact that the data has locally bounded variation.

We have already seen that Riemann data develops a vacuum if condition (2.10) fails. Since the data can be approximated by piecewise constant functions, we thus obtain a condition for the (immediate) formation of a vacuum in general Cauchy data, namely the existence of some $x \in \mathbf{R}$ such that the left and right limits of the data violate (2.10). Thus, assuming the data is locally $B V$, and as usual defining the left and right limits as $\left(v_{0}(x-), u_{0}(x-)\right)$ and $\left(v_{0}(x+), u_{0}(x+)\right)$, respectively, we have the following lemma.

LEMMA 3.1. For any $x \in \mathbf{R}$ such that

$$
u_{0}(x+)-u_{0}(x-) \geq g\left(v_{0}(x-), \infty\right)+g\left(v_{0}(x+), \infty\right)
$$

holds, a vacuum develops immediately at time $t=0+$. In particular, the solution is not stable in $B V_{\text {loc }}$.

Proof. It is well known that the data can be approximated arbitrarily closely by piecewise constants, and the solution in a small neighborhood looks like the solution of the corresponding Riemann problems $[1,3]$. We choose piecewise constant approximations to 
the data which include a jump at $x$ which forms a vacuum in the corresponding Riemann solution.

Indeed, given any $\epsilon>0$, we choose our $\epsilon$-approximation such that there is a jump at $x$, and we take the constant values on either side of $x$ to be exactly $\left(v_{0}(x-), u_{0}(x-)\right)^{t}$ and $\left(v_{0}(x+), u_{0}(x+)\right)^{t}$, respectively. The resulting approximation contains a vacuum with left and right states given by

$$
\begin{aligned}
& u_{-}=u_{0}(x-)+g\left(v_{0}(x-), \infty\right), \\
& u_{+}=u_{0}(x+)-g\left(v_{0}(x+), \infty\right),
\end{aligned}
$$

which are independent of $\epsilon$. We shall see in the next section that the vacuum does not immediately disappear as a result of wave interactions, and thus persists in the limit as $\epsilon \rightarrow 0$.

We shall say that this vacuum, which is an example of a rarefaction vacuum [7], is embedded in the initial data. We shall show that this is the only way that a vacuum can develop from $L^{\infty}$ initial data. That is, a vacuum cannot appear at finite positive times. We will also discuss the possible annihilation of the vacuum.

4. Wave interactions. We now examine Glimm interactions. These are pairwise interactions of elementary waves which we resolve by solving the Riemann problem consisting of the extreme left and right states. We are essentially calculating the asymptotic states which appear after the waves have passed through each other completely. This is exactly what happens inside a 'diamond' [4, 13], and the resulting error estimate is the basis of all pointwise bounds for conservation laws. Here we investigate which wave interactions can give rise to a vacuum state.

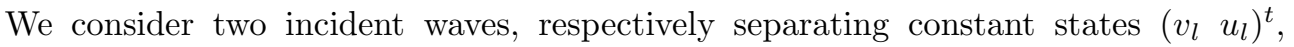
$\left(\begin{array}{ll}v_{m} & u_{m}\end{array}\right)^{t}$ and $\left(v_{r} u_{r}\right)^{t}$. The interaction problem is to resolve the Riemann problem with states $\left(v_{l} u_{l}\right)^{t}$ and $\left(v_{r} u_{r}\right)^{t}$, and to estimate the outgoing waves in terms of the incoming wave strengths. In the present context, since the velocities are given by (2.8), this amounts to solving an identity for the outgoing middle state $v_{*}$.

There are two cases to consider, namely the interaction of two forward (or backward) waves, and the interaction of a forward and backward wave. We first consider forwardforward interactions. By $(2.8)$, across the wave joining $\left(v_{l} u_{l}\right)^{t}$ and $\left(v_{m} u_{m}\right)^{t}$, we have

$$
u_{m}-u_{l}=g\left(v_{m}, v_{l}\right)
$$

while across the wave joining $\left(v_{m} u_{m}\right)^{t}$ and $\left(v_{r} u_{r}\right)^{t}$,

$$
u_{r}-u_{m}=g\left(v_{r}, v_{m}\right) \text {. }
$$

We resolve the Riemann problem according to (2.9), and eliminating $u$ obtain the identity

$$
g\left(v_{l}, v_{*}\right)+g\left(v_{r}, v_{*}\right)=g\left(v_{m}, v_{l}\right)+g\left(v_{r}, v_{m}\right) .
$$

It is well known that in order for there to be any interaction at all, one of the incident waves must be a shock. Since the sound speed is greater behind a shock, we see that after the shock has passed we are further from the vacuum state. Thus if a vacuum is not present in the incident waves, it is certainly not present in the outgoing waves that 
occur in the solutions at later times. We conclude that a vacuum does not develop as a result of the interaction of two waves of the same family.

More precisely, from (2.13) and (2.16), we have

$$
g\left(v_{m}, v_{l}\right)+g\left(v_{r}, v_{m}\right) \leq h\left(v_{l}\right)-h\left(v_{r}\right) \leq \max \left\{g\left(v_{r}, v_{l}\right), g\left(v_{l}, v_{r}\right)\right\},
$$

so that, in (4.1)

$$
v_{*} \leq \max \left\{v_{l}, v_{r}\right\}
$$

and no vacuum develops. Here we have used the fact that $g$ increases in the second variable.

We similarly get an identity for the analysis of the interaction of a forward and backward wave. Indeed, according to (2.8), we have

$$
\begin{aligned}
& u_{m}-u_{l}=g\left(v_{m}, v_{l}\right) \\
& u_{r}-u_{m}=g\left(v_{m}, v_{r}\right),
\end{aligned}
$$

and combining these with (2.9), we get

$$
g\left(v_{l}, v_{*}\right)+g\left(v_{r}, v_{*}\right)=g\left(v_{m}, v_{l}\right)+g\left(v_{m}, v_{r}\right) .
$$

Again we note that if one of the incident waves is a shock, then the sound speed behind the shock is greater, so that the new state $v_{*}$ will be further from the vacuum than one of $v_{l}$ and $v_{r}$. We thus conclude that a vacuum is not the result of a shock and any other wave. Indeed, suppose the left wave is a shock, so that $v_{m}>v_{l}$. Then again

$$
\begin{aligned}
g\left(v_{m}, v_{l}\right)+g\left(v_{m}, v_{r}\right) & <g\left(v_{m}, v_{r}\right) \\
& \leq h\left(v_{r}\right)-h\left(v_{m}\right)<h\left(v_{r}\right)-h\left(v_{l}\right) \\
& \leq \max \left\{g\left(v_{r}, v_{l}\right), g\left(v_{l}, v_{r}\right)\right\},
\end{aligned}
$$

so that $v_{*}<\max \left\{v_{l}, v_{r}\right\}$ and there is no vacuum.

It remains therefore to check the case of two rarefactions interacting. In this case we have $v_{l}, v_{r}>v_{m}$, and (4.2) gives

$$
\begin{aligned}
g\left(v_{m}, v_{l}\right)+g\left(v_{m}, v_{r}\right) & =h\left(v_{l}\right)-h\left(v_{m}\right)+h\left(v_{r}\right)-h\left(v_{m}\right) \\
& \leq h\left(v_{*}\right)-h\left(v_{l}\right)+h\left(v_{*}\right)-h\left(v_{r}\right),
\end{aligned}
$$

so that, since $h$ is increasing,

$$
v_{*}>\max \left\{v_{l}, v_{r}\right\}
$$

and by (2.14), $v_{*}$ must solve the equality

$$
h\left(v_{*}\right)=h\left(v_{l}\right)+h\left(v_{r}\right)-h\left(v_{m}\right) .
$$

Since the integral (1.2) converges, we have $h(\infty)<\infty$, and it is easy to choose the states so that the solvability condition (2.9) fails. Thus our condition for the creation of a vacuum from the interaction of two rarefactions is

$$
h\left(v_{l}\right)+h\left(v_{r}\right)-h\left(v_{m}\right) \geq h(\infty) .
$$

We have seen that the asymptotic state of the interaction of two strong rarefaction waves contains a vacuum as long (4.5) holds. This suggests that we can indeed get vacuums from wave interactions in solutions with general $L^{\infty}$ data. However, we now show 
that the interaction takes place over an unbounded time interval, and so the vacuum does not form in finite time.

4.1. Interaction of rarefactions. We are studying the interaction of forward and backward rarefaction waves. Recall that the forward characteristics are the curves

$$
\frac{d x}{d t}=c(v(x, t))
$$

and $v$ can be used as the parameter across the wave, with $u$ given by (2.4). Thus we can describe any forward rarefaction wave with state $(v u)^{t}$ prescribed along some noncharacteristic curve, as follows.

Given a Cauchy curve parameterized by $\gamma$, that is functions $X, T, v$ and $u$ of $\gamma$ satisfying

$$
\frac{d X}{d \gamma} \neq c(v(\gamma)) \frac{d T}{d \gamma} \quad \text { and } \quad \frac{d u}{d \gamma}=-c(v(\gamma)) \frac{d v}{d \gamma}
$$

and given also some 'target' curve inside the simple wave, $(x, t)$, also parameterized by $\gamma$ and non-characteristic, we have

$$
v(x, t)=v(X, T)=v(\gamma) \text { and } \quad u(x, t)=u(X, T)=u(\gamma)
$$

provided that

$$
x(\gamma)-X(\gamma)=c(v(\gamma))(t(\gamma)-T(\gamma)) .
$$

Now consider the interaction of forward and backward rarefactions, as in Fig. 1. The resultant state once the waves have passed through each other is given by (4.4), and we wish to calculate the region of interaction in the characteristic $(x, t)$-plane. Suppose for the moment that the interaction has a finite solution, $v_{*}<\infty$. Then we can break the plane into three types of regions, namely the regions in which $v$ is constant, the simple rarefaction waves, and the interaction region [3]. The boundaries between these different regions are characteristic curves of the appropriate family, and we will now describe these curves.

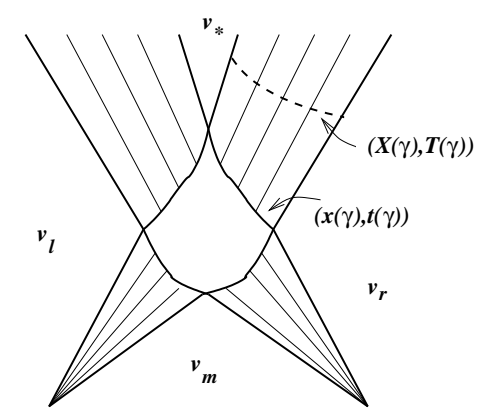

Figure 1. Interaction of rarefactions

It is clear that the curves bounding the constant states are forward and backward characteristics which are straight lines. We now describe the boundary of the interaction regions, which are also characteristics [3]. Suppose we are to calculate the boundary through which the forward rarefaction leaves the interaction region, which is a backward characteristic. Choose a curve which crosses the rarefaction and is non-characteristic, say 
$(X(\gamma), T(\gamma))$. Along this curve, the state is $(v(\gamma) u(\gamma))^{t}$. Actually, all we need is the dependence $c(\gamma)=c(v(\gamma))$. Now we describe the boundary with the interaction region by $(x(\gamma), t(\gamma))$, also parameterized by $\gamma$, and such that (4.6) holds.

Since the boundary of the interaction region is a backward characteristic, we have

$$
\frac{d x}{d \gamma}=-c(\gamma) \frac{d t}{d \gamma}
$$

From (4.6), we have

$$
x(\gamma)-c(\gamma) t(\gamma)=H(\gamma) \equiv X(\gamma)-c(\gamma) T(\gamma),
$$

where we regard $H(\gamma)$ as a known function of $\gamma$, which can be taken to be decreasing, $H^{\prime}(\gamma) \leq 0$, because the curve $(X, T)$ is non-characteristic and the outgoing wave is a rarefaction. Differentiating and using (4.7), we get

$$
-c(\gamma) \frac{d t}{d \gamma}-\frac{d}{d \gamma}(c(\gamma) t(\gamma))=H^{\prime}(\gamma)
$$

a first order linear differential equation for the function $t(\gamma)$. Solving for $t(\gamma)$, we get

$$
\sqrt{c(\gamma)} t(\gamma)-\sqrt{c\left(\gamma_{0}\right)} t\left(\gamma_{0}\right)=\int_{\gamma_{0}}^{\gamma} \frac{-H^{\prime}}{2 \sqrt{c}} d \gamma
$$

We have the following theorem.

THEOREM 3. A vacuum cannot form from wave interactions in finite time. Thus any vacuum that appears in the solution of (1.1) must be present in the initial data. In particular, if the initial velocity is continuous, there is no vacuum in the solution.

Proof. We have seen that the only possible way for a vacuum to form is from the interaction of two rarefactions. We calculate the boundary of the interaction region as above, for interactions which yield a finite middle state $v_{*}$. Since the rarefaction curves piece together continuously, by varying the entering states, we can change $v_{*}$. If we parameterize the outgoing wave with $\gamma$ increasing across the wave from ahead to behind, from (4.8) we have

$$
\sqrt{c\left(v_{*}\right)} t\left(v_{*}\right)-\sqrt{c\left(v_{r}\right)} t\left(v_{r}\right) \geq 0,
$$

where $t(v)$ is the time at which state $v$ emerges from the interaction region. Now the vacuum is characterized by $c(v)=0$, and taking the limit as $c\left(v_{*}\right) \rightarrow 0$, we must have $t\left(v_{*}\right) \rightarrow \infty$.

No vacuum can develop in finite time: thus any vacuum must be present in the data, and so appear at an $x$ at which condition (3.4) holds. In particular, if the initial velocity $u_{0}$ is continuous, we have $u_{0}\left(x_{-}\right)=u_{0}\left(x_{+}\right)$for each $x$, and (3.4) does not hold anywhere.

If the rarefaction is a centered wave, then we can take $H(\gamma)$ to be a constant, so there is no right hand side in (4.8). In this case, the lower boundary of the interaction zone is easily seen to be a hyperbola. Moreover, this is the extreme case, and for general rarefactions the right hand side of (4.8) is strictly positive, so the characteristic moves into the rarefaction more slowly. 
5. Shocks and the vacuum. We now discuss the effects of shock waves on the vacuum. This question has been considered by Liu and Smoller [7] in the Eulerian formulation, which is equivalent to the $p$-system [10]. As above, we start by analyzing the interaction of a shock wave with a rarefaction. For brevity we consider a forward shock interacting with a backward rarefaction centered at the origin. Thus suppose that $\left(v_{l} u_{l}\right)^{t}$ is joined to $\left(v_{m} u_{m}\right)^{t}$ by a shock, and $\left(v_{m} u_{m}\right)^{t}$ to $\left(v_{r} u_{r}\right)^{t}$ by a rarefaction.

The emergent state $\left(v_{*} u_{*}\right)^{t}$ leaving the interaction is given by (4.2). By our choice of incident waves, we have

$$
v_{l}<v_{m}<v_{r}
$$

so by $(2.13),(4.2)$ reduces to

$$
h\left(v_{*}\right)=h\left(v_{l}\right)+h\left(v_{r}\right)-h\left(v_{m}\right)+\frac{1}{2}\left(\theta\left(v_{m}, v_{l}\right)-\theta\left(v_{r}, v_{*}\right)\right)-\frac{1}{2} \theta\left(v_{l}, v_{*}\right) .
$$

We now claim also

$$
v_{l}<v_{*}<v_{r}
$$

so that the outgoing waves are a backward rarefaction and forward shock, as would be expected, and the last term in (5.1) vanishes. Indeed, (4.3) gives $v_{*}<v_{r}$, and assuming $v_{*} \leq v_{l}$, we get the contradiction

$$
\begin{aligned}
0 & \geq h\left(v_{*}\right)-h\left(v_{l}\right)+h\left(v_{m}\right)-h\left(v_{r}\right) \\
& =\frac{1}{2}\left(\theta\left(v_{m}, v_{l}\right)-\theta\left(v_{r}, v_{l}\right)+\theta\left(v_{r}, v_{l}\right)-\theta\left(v_{r}, v_{*}\right)-\theta\left(v_{l}, v_{*}\right)\right) \\
& >0
\end{aligned}
$$

where we have used (2.15).

Since the backward waves are rarefactions, we can piece them together and the state $v_{*}$ changes smoothly with $v_{r}$. We can thus trace the path of the shock wave as it passes through the rarefaction, as we did for characteristics in the previous section. We assume a backward rarefaction centered at the origin (so $H \equiv 0$ ), and it is convenient to use the sound speed $c(v)$ as the parameter. Then if the path of the shock wave inside the rarefaction is $(x, t)$, we have

$$
x(c)+c t(c)=0,
$$

where $c$ decreases from $c_{m}=c\left(v_{m}\right)$ to $c_{r}=c\left(v_{r}\right)$. On the other hand, the shock path is given by

$$
\frac{d x}{d c}=\sigma(c) \frac{d t}{d c}
$$

where $\sigma(c)$ is the absolute shock speed,

$$
\sigma(c)=\sigma\left(v, v_{\#}\right) .
$$

Here $v=v(c)$ is the ahead state inside the centered rarefaction, and $v_{\#}$ is the behind state, given by

$$
g\left(v_{l}, v_{\#}\right)+g\left(v, v_{\#}\right)=g\left(v_{m}, v_{l}\right)+g\left(v_{m}, v\right) .
$$

Note that as $c$ varies from $c_{m}$ to $c_{r}, v_{\#}$ varies smoothly from $v_{l}$ to $v_{*}$. 
As above, we regard $\sigma(c)$ as a known function of $c$, differentiate (5.2), and use (5.3) to get the differential equation

$$
(\sigma+c) \frac{d t}{d c}+t(c)=0
$$

Solving, we get

$$
\log t(c)-\log t\left(c_{m}\right)=\int_{c}^{c_{m}} \frac{d c}{\sigma+c} .
$$

We have thus calculated the states behind the shock, $v_{\#}$, and the path of the shock, (5.4), (5.2). This together with our earlier description of a simple wave yields an exact description of the global solution provided no other interactions take place. In particular, if the shock is initially a finite distance away from the vacuum, the vacuum persists for a finite time.

We now examine what happens as the shock approaches the vacuum. First, note that our strict inequality

$$
v_{r}>v_{*},
$$

implies that as long as $v_{r}$ remains finite, the shock persists. Thus we consider the limit $v_{r} \rightarrow \infty$ (or equivalently $c_{r} \rightarrow 0$ ). From (5.4), the shock will meet the vacuum in finite time provided the integral converges,

$$
\int_{0}^{c_{m}} \frac{d c}{\sigma+c}<\infty
$$

The boundedness of this integral follows from the observation that

$$
\sigma\left(v, v_{\#}\right)=\frac{g\left(v, v_{\#}\right)}{v_{\#}-v},
$$

which is (2.12). Then, still using $c$ as the parameter, we have

$$
\int \frac{d c}{\sigma+c}=\int \frac{\left(v_{\#}-v\right) d c}{g\left(v, v_{\#}\right)+c\left(v_{\#}-v\right)} .
$$

Now our assumptions on $c$, namely

$$
c^{\prime}(v)<0 \text { and } \int_{1}^{\infty} c(v) d v<\infty,
$$

imply that $c \cdot v \rightarrow 0$, and an integration by parts yields also

$$
\int_{0}^{c} v(c) d c=\left.c \cdot v\right|_{0} ^{c}-\int_{\infty}^{v} c d v<\infty .
$$

Finiteness of the integral now follows since the denominator tends to

$$
\lim _{v_{r} \rightarrow \infty} g\left(v_{r}, v_{*}\right)=-k<0 .
$$

Indeed, using (2.13) in (5.1), we have

$$
\begin{aligned}
2 g\left(v_{r}, v_{*}\right) & =2\left(h\left(v_{l}\right)-h\left(v_{m}\right)\right)+\theta\left(v_{m}, v_{l}\right)+\theta\left(v_{r}, v_{*}\right) \\
& \leq 2\left(h\left(v_{l}\right)-h\left(v_{m}\right)\right)+\theta\left(v_{m}, v_{l}\right)<0 .
\end{aligned}
$$


As in the previous section, we get the same results for a shock entering a rarefaction which is not centered. In this case, our differential equation has a right hand side $H^{\prime}(\gamma)$, but the integral remains finite.

We have shown that the shock disappears in finite time as it meets the vacuum. In continuing the solution beyond the time the shock meets the vacuum, we must resolve the Riemann problem with left state $\left(v_{*} u_{*}\right)^{t}$ and right state that on the opposite side of the vacuum, $\left(v_{+} u_{+}\right)^{t}$, taking into account the delta function that is present. Stability considerations imply that the vacuum cannot be immediately annihilated by a single shock interaction, i.e. the specific volume does not immediately become finite. Rather, the shock weakens the vacuum, corresponding to a decrease in the coefficient $w(t)$ in $(3.2)$. The effect of this in Eulerian coordinates is to slow down the expansion of the vacuum, possibly changing it from a rarefaction vacuum to a compression vacuum which will eventually extinguish itself [7].

An exception occurs when the condition for a vacuum holds identically, so the left and right limits of (3.5) are equal,

$$
u_{-}=u_{+}, \quad \text { so that } \quad w(t)=0,
$$

and the vacuum has zero strength (or zero width in physical coordinates). This is a special degenerate case of a vacuum extinguishing itself, and the vacuum will disappear when it is met by a shock of finite strength.

6. Conclusions. We have shown that solutions to the $p$-system with continuous data do not form a vacuum in finite time. Nevertheless, if we allow discontinuities in the data, a vacuum may form. The condition to check whether or not the vacuum will form is explicit, and is particularly simple in the gas of $\gamma$-law gases. Thus it is readily checked and computable.

The main difficulty in proving a constructive existence proof for solutions of systems of conservation laws is obtaining estimates which allow one to pass to the limit. These are typically $B V$ estimates, which are inappropriate near the vacuum. Indeed, we have seen that the Riemann problem is not stable in $B V_{\text {loc }}$. Liu and Smoller have shown that Glimm's basic interaction estimate, which is the building block of $B V$ estimates, fails near the vacuum [7]. Indeed, this is clear here because finite rarefaction waves can fail to pass through each other completely, so the interaction cannot be approximated by a linear interaction.

Our approach to the problem of finding global estimates is traditional in terms of studying wave interactions. However, we take the point of view that one should look at exact results of interactions, rather than at asymptotic descriptions as has thus far been the case $[4,9,13]$. This point of view has been made possible by the development of front tracking approximations [1]. Indeed, the additive structure of rarefactions and the fact that other waves have zero width fit well with the front tracking approximations. By carefully approximating rarefactions and their interactions, one can obtain exact descriptions of the evolution of the states, so that only the trajectories in the plane are approximations.

We outline briefly an approach to obtaining global estimates, pursued further in [11]. 
First, identify the positions where a vacuum will appear in the data (which do not evolve as we are in Lagrangian coordinates). Next, take some compact set $K$ which does not meet any of these vacuums. We now search for a $B V$ bound on this compact set. To do so, identify all the waves in the solution initially. Now, since the result of any interaction inside $K$ is finite, we formally identify all pairs of waves which will interact inside $K$, and put the corresponding exact expression for the interaction into a potential. Since $K$ is compact, this potential will be finite and independent of the size of the approximation parameter, and thus we can pass to the limit. We remark here that in a front tracking approximation, because we have a $2 \times 2$ system, the number of waves is conserved and there are no resonances due to nonvanishing Lie brackets [12]. Also, because $K$ is compact and away from the vacuum states, a domain of dependency should exist which also avoids the vacuum. One problem here, however, is if there are many shocks which enter a vacuum, causing it to eventually be extinguished, strong shocks may appear [7], which would then be reflected back into $K$. Thus a better understanding of how a 'compression vacuum' is extinguished is needed. Note however, that when a vacuum is formed from $L^{\infty}$ data, it is a rarefaction vacuum, and so a minimum amount of shock strength is needed to convert it into a compression vacuum, and the vacuum will persist for some finite time.

\section{References}

[1] A. Bressan, Hyperbolic Systems of Conservation Laws: The One-dimensional Cauchy Problem, Oxford Lecture Ser. Math. Appl. 20, Oxford Univ. Press, Oxford, 2000.

[2] C. M. Dafermos, Hyperbolic Conservation Laws in Continuum Physics, Grundlehren Math. Wiss. 325, Springer, Berlin, 2000.

[3] R. DiPerna, Singularities of solutions of nonlinear hyperbolic systems of conservation laws, Arch. Rat. Mech. Anal. 60 (1975/76), 75-100.

[4] J. Glimm, Solutions in the large for nonlinear hyperbolic systems of equations, Comm. Pure Appl. Math. 18 (1965), 697-715.

[5] J. Glimm and P. D. LAx, Decay of solutions of systems of nonlinear hyperbolic conservation laws, Memoirs Amer. Math. Soc. 101 (1970).

[6] P. D. LAx, Hyperbolic systems of conservation laws, II, Comm. Pure Appl. Math. 10 (1957), $537-566$.

[7] T.-P. LiU and J. A. Smoller, On the vacuum state for the isentropic gas dynamics equations, Adv. in Appl. Math. 1 (1980), 345-359.

[8] J. SMoller, On the solution of the Riemann problem with general step data for an extended class of hyperbolic systems, Michigan Math. J. 16 (1969), 201-210.

[9] J. Smoller, Shock Waves and Reaction-Diffusion Equations, Grundlehren Math. Wiss. 258, Springer, New York, 1982.

[10] D. WAGNER, Equivalence of the Euler and Lagrangian equations of gas dynamics for weak solutions, J. Differential Equations 68 (1987), 118-136.

[11] R. Young, The p-system with large data, in preparation.

[12] R. Young, On elementary interactions for hyperbolic conservation laws, Preprint, 1993. 
[13] R. Young, Sup-norm stability for Glimm's scheme, Comm. Pure Appl. Math. 46 (1993), 903-948.

[14] R. Young, Nonstrictly hyperbolic waves in elasticity, J. Differential Equations (to appear).

[15] R. Young, The p-system I: The Riemann problem, in: The Legacy of the Inverse Scattering Transform in Applied Mathematics, Contemp. Math. 301, Amer. Math. Soc., Providence, 2002, 219-234.

[16] R. Young, Wave interactions in nonlinear elastic strings, Arch. Rat. Mech. Anal. 161 (2002), 65-92. 